\title{
Polymer nanofiber-based microchips for EGFR mutation analysis of circulating tumor cells in lung adenocarcinoma
}

This article was published in the following Dove Press journal: International Journal of Nanomedicine

\author{
Wenting Jiangl,* \\ Han Wangl,* \\ Yongmei Cuil,* \\ Yiyan Lei ${ }^{2}$ \\ Yuefeng Wang' \\ $\mathrm{Di} \mathrm{Xu}^{3}$ \\ Neng Jiang' \\ Yangshan Chen' \\ Yu Sun' \\ Yang Zhang ${ }^{4}$ \\ Jessica $\mathrm{Cao}^{5}$ \\ Zunfu Ke
}

'Department of Pathology, The First Affiliated Hospital, Sun Yat-sen University, Guangzhou, Guangdong, China; ${ }^{2}$ Department of Thoracic Surgery, The First Affiliated Hospital, Sun Yat-sen University, Guangzhou, Guangdong, China; ${ }^{3}$ Department of Thoracic Surgery, The Central Hospital of Wuhan, Jiang'an District, Wuhan, Hubei, China; ${ }^{4}$ Biomedical Engineering, The University of Texas at El Paso, El Paso, TX, USA; ${ }^{5}$ Cumming School of Medicine, University of Calgary, Calgary, AB, Canada

*These authors contributed equally to this work

Correspondence: Zunfu Ke Department of Pathology, The First Affiliated Hospital, Sun Yat-sen University, Number 58, ZhongShan Second Road, Guangzhou, Guangdong 510080 , China

Tel/fax +86208733 1780

Email kezunfu@mail.sysu.edu.cn
Background: Circulating tumor cells (CTCs) detection, an approach considered to be "liquid biopsy", is a potential alternative method in clinical use for early diagnosis of solid tumor progression.

Methods: In this study, we developed a poly (lactic-co-glycolic acid) (PLGA) - nanofiber (PN)NanoVelcro chip as an efficient device for simple and rapid capture of CTCs from peripheral blood. We evaluated the device performance by assessing the capture efficiency and purity. Single CTC was isolated via laser microdissection system for subsequent genetic analysis, with an aim to find the concordance of epidermal growth factor receptor (EGFR) mutations between tumor tissue and CTCs.

Results: PN-NanoVelcro chip exhibits great performance in capture efficiency and high purity. The genetic analysis results showed that most EGFR mutation in tumor tissue could also be detected in CTCs.

Conclusion: Compared to computed tomography image results, CTC detection can be implemented throughout the course of diseases and provides an accurate and earlier diagnosis of tumor progression, which make it possible for patients to acquire suitable and timely treatment.

Keywords: PN-NanoVelcro chip, CTCs, EGFR mutation, prognosis, lung adenocarcinoma

\section{Introduction}

Lung cancer is the main cause of cancer-related death due to its late prognosis and poor therapy, leading to 1.6 million deaths annually. ${ }^{1}$ Non-small cell lung cancer (NSCLC), accounting for $\sim 80 \%$ of lung cancers, can also be divided into two subtypes: adenocarcinoma and squamous cell carcinoma. Lung adenocarcinoma (LADC), representing about one-half of all lung cancers, is the most frequent histologic subtype of NSCLC. ${ }^{2}$ Although significant advancement has been achieved in chemotherapy, targeted therapy and surgical technique, the average 5-year survival rate of NSCLC was just $18 \%$ due to its late diagnosis and lack of timely and efficient treatment. ${ }^{3}$ As known to all, metastasis mostly contributes to the progression of cancer, so early detection of metastasis is of great significance for early intervention and individual treatment.

During the progression of cancer metastasis, circulating tumor cells (CTCs) are shed from the solid primary tumor, enter into the blood stream and travel to remote tissues. In recent years, numerous researches have demonstrated that CTC analysis could be used in detecting early tumor progression of various kinds of cancer, including breast, colorectal, prostate cancers and so on. ${ }^{4-7}$ Thus, CTC analysis is now regarded as "liquid biopsy" and has been used in clinical application to predict the clinical 
outcomes and monitor treatment responses throughout the course of diseases. Although Cell Search ${ }^{\mathrm{TM}}$ had been allowed by the US Food and Drug Administration to detect CTCs in the clinical application in 2004, it was still constrained by its low sensitivity in epithelial cell adhesion molecule (EpCAM)-negative cases. ${ }^{8}$ With the development of technology, various approaches have been found for capturing rare CTCs in peripheral blood more conveniently and efficiently, including capture agent-labeled magnetic beads, ${ }^{9,10}$ microfluidic device ${ }^{11}$ and microfilter device. ${ }^{12,13}$ However, due to the extremely low number of CTCs in peripheral blood and limitation of technology, enrichment and detection approaches still need to be further improved for the sake of identifying and characterizing CTCs.

Herein, we developed polymer nanofiber-based microchips to capture CTCs and implemented Laser Microdissection System to isolate single CTC, followed by subsequent genetic analysis. Recent advances in amplification refractory mutation system polymerase chain reaction (ARMS-PCR) have made it possible to investigate the genomic alterations in CTCs. ${ }^{14}$ With the assistance of polymer nanofiber-based microchips and genetic analysis technologies, CTC detection could be used in timely detection of progression of cancer.

Epidermal growth factor receptor (EGFR), a member of the ErbB family, is a transmembrane tyrosine kinase receptor. ${ }^{15}$ Accumulating researches had demonstrated that EGFR mutation occurred frequently in non-smoking female Asian patients with LADC. ${ }^{16-18}$ Gefitinib, an EGFR tyrosine kinase inhibitor (TKI), is widely used in clinical application as a molecular-targeting drug in the treatment of NSCLC. Unlike those patients with EGFR wild-type tumors, patients with EGFR mutations (exon 21 L858R mutation or exon 19 deletions) benefit much from EGFR-TKI treatment. ${ }^{19}$ However, although patients responded well to EGFR-TKI treatment initially, they would finally develop resistance and about $50 \%$ of this resistance could be attributed to T790M mutation, the emergence of EGFR-resistant mutation. ${ }^{20}$ Currently, tumor tissues obtained from surgery are used as a standard sample for EGFR mutation detection in the clinical practice, but it still has several limitations. ${ }^{21}$ Herein, our present study aims to develop CTC analysis as a promising, noninvasive alternative for EGFR mutation detection in clinical application.

\section{Materials and methods}

Polymer nanofiber-based microchips preparation and surface coating

PLGA was fabricated onto the substrates by using electrospinning technology (Figure1A). The polymer nanofiber-based microchip comprises two functional components, including an overlaid polydimethylsiloxane (PDMS) chaotic mixer and a transparent poly(lactic-co-glycolic acid) (PLGA) nanofiber (PN)-NanoVelcro substrate (Figure 1B). PLGA, a polymeric material with extremely good compatibility, was electrospun onto laser capture microdissection (LCM) glass slide. PLGA nanofibers were covalently conjugated with streptavidin through 1-ethyl-3-(3-dimethylaminopropyl)carbodiimide/ $N$-hydroxy-succinimide chemistry, which was employed to activate the carboxylic acid groups of PLGA. Then, streptavidin was conjugated with antiEpCAM, which especially binds to EpCAM on the CTCs (Figure 1C).

\section{Patient characteristics and clinical samples}

A total of 72 NSCLC patients were enrolled in the study, consisting of 27 males $(37.50 \%)$ and 45 females $(62.50 \%)$, including 18 smokers and 54 non-smokers. The patients could be divided into four groups according to the clinical stages. Other characteristics are clearly listed in Table 1. Written informed consent was obtained from every patient.

Blood samples and tissue samples from both patients with lung cancer and healthy donors were recruited from the First Affiliated Hospital of Sun Yat-sen University and were used to perform subsequent studies. The blood samples were drawn into vaccutainer collection tubes containing EDTA and processed within $12 \mathrm{~h}$, while the tissue samples were stored in liquid nitrogen. This study was approved by the institutional ethics committee of the First Affiliated Hospital of Sun Yat-sen University.

\section{CTCs enrichment procedure}

One milliliter of whole blood sample was initially subjected to red blood cell lysis buffer followed by $20 \mathrm{~min}$ of incubation at room temperature until the red blood cells were completely lysed. Then the supernatant was removed after centrifugation at $200 \times g$ at $4^{\circ} \mathrm{C}$ for $5 \mathrm{~min}$. After centrifugation, the cell pellets were resuspended by the same volume of $1 \times$ PBS. SK-BR-3 cells (American Type Culture Collection, Manassas, VA, USA), stained with fluorescent markers from Circulating Tumor Cell Control Kit (Cell Search, San Diego, CA, USA), were used to spike into the suspension solution as artificial CTC blood sample. The same number of SK-BR-3 cells was spiked into another $1 \mathrm{~mL}$ of whole blood to mimic the CTCs in peripheral blood. CTC enrichment was implemented through polymer nanofiber-based microchips at a flow rate of $1 \mathrm{~mL} / \mathrm{h}$ at room temperature for $1 \mathrm{~h}$. 
A
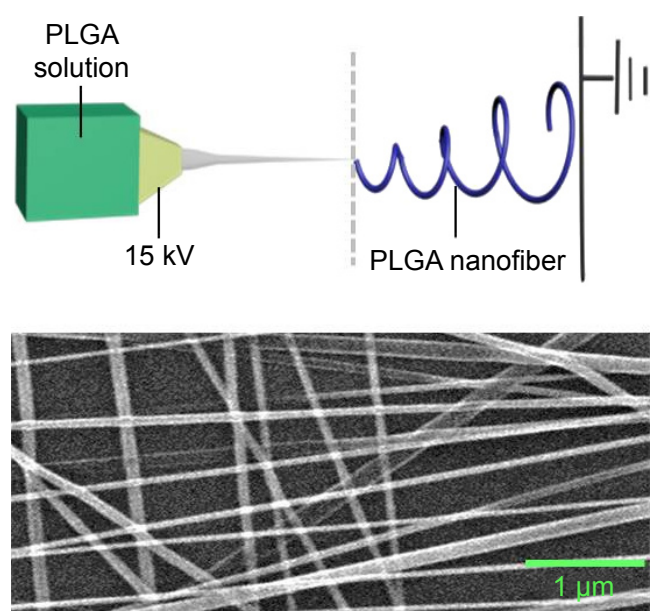

C

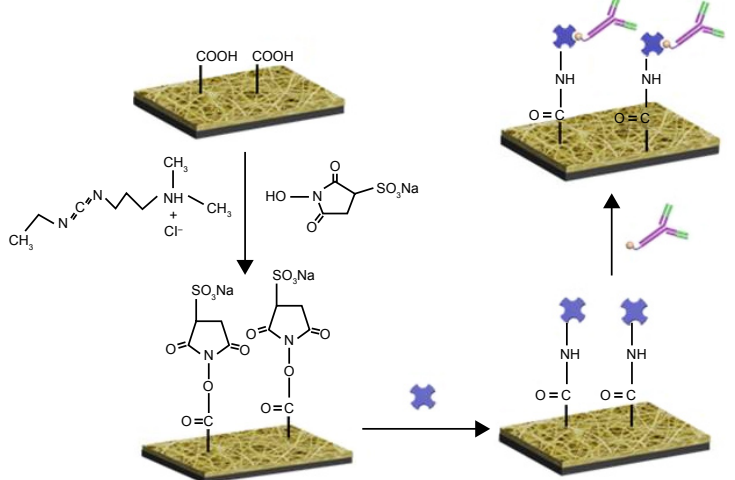

B

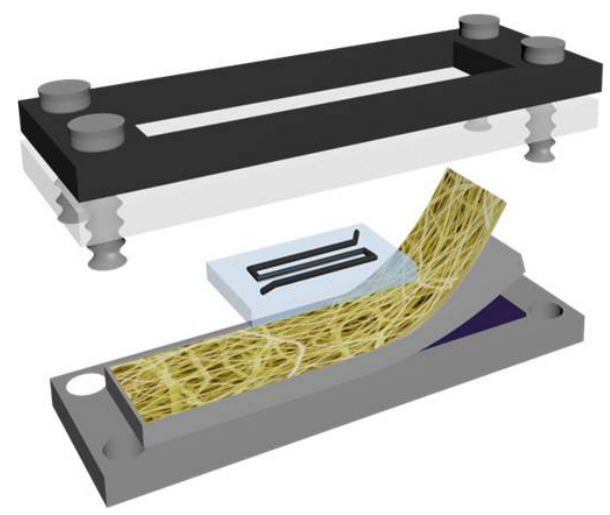

D

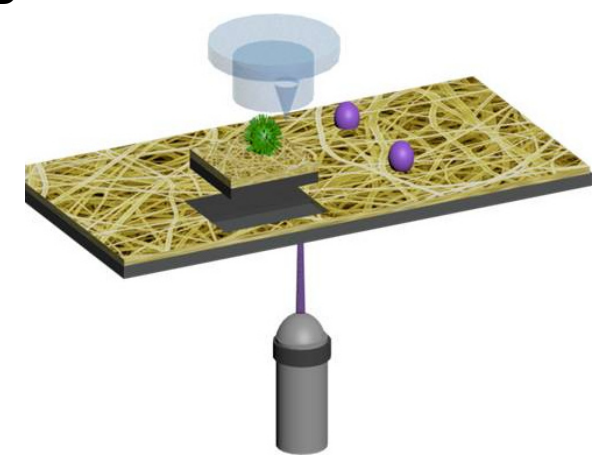

Figure I The configuration of PLGA nanofiber microchip for the high-purity isolation of CTCs.

Notes: (A) A transparent PN-NanoVelcro substrate was prepared by depositing electrospun PLGA nanofibers onto a commercial LMD glass slide (with a pre-deposited $1.2 \mu \mathrm{m}$ thick PPS membrane). The SEM image of the electrospun PLGA nanofibers is shown. (B) A custom-designed chip holder is used to sandwich a microchip that is composed of an overlaid PDMS chaotic mixer chip. (C) Schematic representation of the chemistry of EpCAM-modified PLGA for cancer cell capture applications. (D) Single CTC isolation by laser microdissection.

Abbreviations: CTCs, circulating tumor cells; EpCAM, epithelial cell adhesion molecule; LMD, laser microdissection; PLGA, poly(lactic-co-glycolic acid); SEM, scanning electron micrograph.

\section{Immunostaining and imaging}

NCl-H1975 cells, a cell line of LADC which was purchased from Shanghai Institute of Cell Biology, were spiked in whole blood for the examination of performance of polymer nanofiber-based microchips. After washing with $1 \times$ PBS for three times, the captured cells were fixed with $4 \%$

Table I Clinical characteristics of 72 NSCLC patients

\begin{tabular}{|c|c|c|c|}
\hline & All cases (\%) & & All cases (\%) \\
\hline \multicolumn{2}{|l|}{ Age (years) } & \multicolumn{2}{|c|}{ Gender } \\
\hline$\leq 50$ & $34(47.22)$ & Male & $27(37.50)$ \\
\hline$>50$ & $38(52.78)$ & Female & $45(62.50)$ \\
\hline \multicolumn{2}{|l|}{ Differentiation degree } & \multicolumn{2}{|c|}{ Clinical stage } \\
\hline Well differentiated & $18(25.00)$ & I & $8(11.11)$ \\
\hline Moderately differentiated & $33(45.83)$ & II & II (I5.28) \\
\hline \multirow[t]{2}{*}{ Poorly differentiated } & $21(29.17)$ & III & $38(52.78)$ \\
\hline & & IV & $15(20.83)$ \\
\hline \multicolumn{2}{|l|}{ Vital status (at follow-up) } & \multicolumn{2}{|c|}{ Smoking } \\
\hline Alive & $53(73.61)$ & Yes & $18(19.36)$ \\
\hline Death & $19(26.39)$ & No & $54(80.65)$ \\
\hline
\end{tabular}

Abbreviation: NSLC, non-small cell lung cancer. paraformaldehyde for 20 min, permeated with $0.1 \%$ triton X-100 in $1 \times$ PBS for 5 min and then blocked with 5\% bovine serum albumin for half an hour. Subsequently, the cells were stained with mouse anti-human pan cytokeratin (Abcam, Cambridge, UK) and rabbit anti-human CD45 (CST, MA, USA) overnight at $4{ }^{\circ} \mathrm{C}$, followed by washing with $1 \times \mathrm{PBS}$ for three times. After washing with $1 \times$ PBS, the cells were stained with fluorescein isothiocyanate-labeled goat-anti-mouse secondary antibody and tetramethylrhodamine-labeled goat-anti-rabbit secondary antibody for $1 \mathrm{~h}$ at room temperature. After thorough washing, 4,6-diamino-12-phenyl indole (DAPI) was used for nuclear staining. CTCs were identified under a fluorescence microscope.

\section{Laser microdissection and CTC mutation analysis}

After confirming the locations of the CTCs by immunostaining, the microchip was placed on the LCM microscope (ArcturusXT ${ }^{\mathrm{TM}}$ ), which is used for isolating a single cell. ${ }^{12}$ 
Then, single CTC was dissected by laser (Figure 1D) and collected in $200 \mu \mathrm{L}$ tube caps, with $10 \mu \mathrm{L}$ lysis solution (40 $\mu \mathrm{g} / \mathrm{mL}$ proteinase $\mathrm{K}$ in PBS, $0.5 \%$ Triton X-100) on the inside surface of it. ${ }^{14,22}$ The purified CTCs were analyzed through subsequent targeting sequencing and whole genome amplification (WGA).

\section{Concordance of EGFR mutations in primary tissues and CTCs}

We recovered single CTC through white blood cell (WBC) depletion on the LCM device and analyzed EGFR mutations in the isolated CTCs in comparison with its primary tumor. Targeted sequencing technology was used to find out the similarity of EGFR mutations in CTCs and primary tissues. KAPA Hyper Prep Kit (Kapa Biosystems, Wilmington, MA, USA) and the SureSelect Target Enrichment System (Agilent Technologies, Santa Clara, CA, USA) were used following the instructions to prepare sequencing libraries for targeted sequencing analysis. Then, targeted sequencing was performed on an Illumina HiSeq 2000 (Illumina, San Diego, CA, USA), and the results were analyzed by the Genomics Workbench software (Agilent Technologies).

\section{Genomic DNA extraction and genetic analysis}

Genomic DNA of primary tissue and CTCs were extracted by QIAamp DNA Mini Kit (Qiagen, Hilden, Germany) according to the manufacturer's instructions. Genomic DNA was eluted with $10 \mu \mathrm{L}$ nuclease-free water for subsequent analysis. $3 \mu \mathrm{L}$ of the extracted DNA was used for WGA using the Ampli1" TM WGA Kit (Silicon Biosystems, Zona Artigianale BO, Italy) according to manufacturer's protocol, and $2 \mu \mathrm{L}$ products were analyzed for quality control by utilizing the Ampli1 QC Kit (Silicon Biosystems). ${ }^{23}$

Polymerase chain reaction (PCR) was used to separately amplify EGFR exons 20 and 21 fragments containing the mutation sites. The PCR steps were performed in a $25 \mu \mathrm{L}$ reaction volume, containing $2 \mu \mathrm{L}$ WGA products, $2 \mu \mathrm{L}$ sense primer $(10 \mu \mathrm{mol} / \mathrm{L}), 2 \mu \mathrm{L}$ antisense primer $(10 \mu \mathrm{mol} / \mathrm{L})$, $2.5 \mu \mathrm{L} 10 \times$ PCR buffer, $2.5 \mu \mathrm{L} \mathrm{MgCl}_{2}(25 \mathrm{mmol} / \mathrm{L}), 1 \mu \mathrm{L}$ deoxyribonucleotide triphosphate $(10 \mathrm{nmol} / \mathrm{L}), 0.5 \mu \mathrm{L}$ Taq-polymerase and $12.5 \mu \mathrm{L} \mathrm{ddH}_{2} \mathrm{O}$. The thermocycling conditions for exon 20 and exon 21 were as follows: 1 cycle of $95^{\circ} \mathrm{C}$ for $10 \mathrm{~min}, 40$ cycles of $94^{\circ} \mathrm{C}$ for $30 \mathrm{~s}$ and $58^{\circ} \mathrm{C}$ for $1 \mathrm{~min}$, followed by $4^{\circ} \mathrm{C}$ hold. PCR products were detected by gel electrophoresis on a $1 \%$ agarose gel.

L858R mutation in exon 20 and T790M mutation in exon 21 were detected using ARMS-PCR. AMPS-PCR was performed according to the protocol of the Human EGFR Gene Mutation Quantitative Detection Kit (fluorescent PCR; Beijing ACCB Biotech Ltd, Beijing, China). Also, the thermal cycling conditions were as follows: $95^{\circ} \mathrm{C}$ for $10 \mathrm{~min}$, followed by 40 cycles of $95^{\circ} \mathrm{C}$ for $15 \mathrm{~s}$ and $60^{\circ} \mathrm{C}$ for $60 \mathrm{~s}$, and fluorescent signals were collected at the end of each cycle of $60^{\circ} \mathrm{C}$ for $60 \mathrm{s.}^{24}$

\section{Relationship between T790M and gefitinib-induced acquired resistance}

Computed tomography (CT) was performed to observe tumor progression, while ARMS-PCR was performed to detect the mutations of L858R and T790M during continuous gefitinib treatment, aiming to find out the relationship between specific EGFR mutation and gefitinib-induced acquired resistance.

\section{Results}

\section{Fabrication of PLGA nanofibers}

PLGA was fabricated onto the substrates by using electrospinning technology. As shown in the scanning electron micrograph, the nanofibers were smooth, randomly orientated and uniform, without any defects of curved and cross-linked nanofibers by fabricating at $15 \mathrm{~cm}$ receiving distance, using $0.5 \mathrm{~mL} / \mathrm{h}$ as the feeding rate (Figure $1 \mathrm{~A}$ ).

\section{Cell capture in the nanofiber-embedded microchip}

During the optimization test of electrospinning time, we discovered that $3 \mathrm{~h}$ of deposition led to better CTC capture efficiency (Figure 2A). The $3 \mu \mathrm{m}$ thick PLGA nanofibers were fabricated through electrospining for $3 \mathrm{~h}$ for the following experiment. Apart from the electrospinning time, flow rate was another significant condition that could not be ignored. Flow rates ranging from 0.1 to $2 \mathrm{~mL} / \mathrm{h}$ were used to optimize the flow rate for subsequent studies. As shown, $0.5 \mathrm{~mL} / \mathrm{h}$ flow rate could achieve the best capture performance (Figure 2B). Also, the distribution of cells was mainly focused on the first four microchannels, accounting for about $78 \%$ (Figure 2C). In addition, PN-NanoVelcro chip design had an effect on the capture efficiency. As observed, the capture efficiency of PN-NanoVelcro chips with herringbone and modified with EpCAM could reach $~ 90 \%$, much higher than PN-NanoVelcro chips with no herringbone, PLGA film coated with anti-EpCAM and PN-NanoVelcro chips with no anti-EpCAM (Figure 2D). In order to evaluate the capture efficiency for different CTC numbers, different numbers of SK-BR-3 cells prestained with DIO, ranging from 10 to 1,000 , were spiked into both PBS and whole 

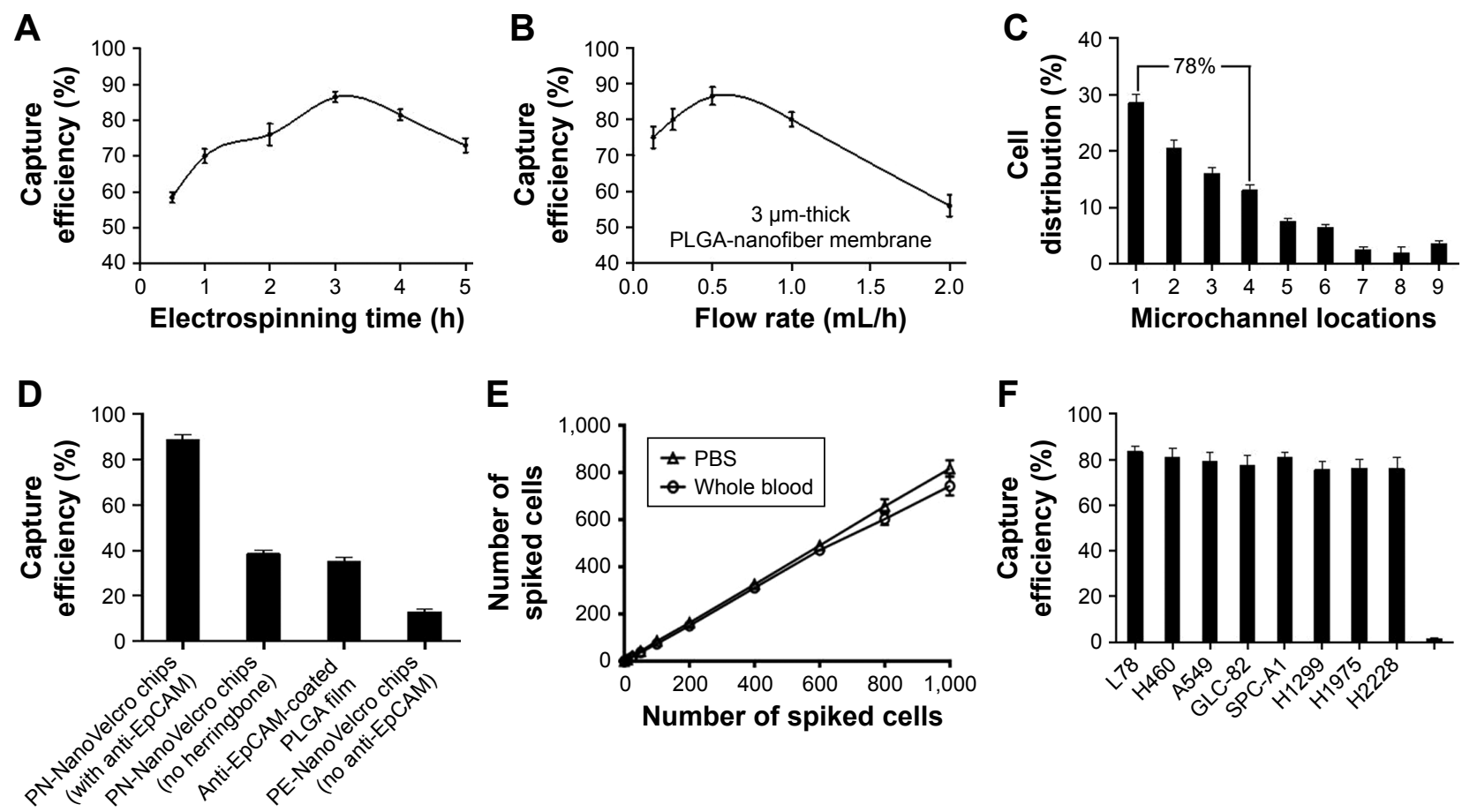

Figure 2 Optimization and validation of the PN-NanoVelcro chip using circulating tumor cell control kit and various cell lines.

Notes: (A) The cell capture efficiency of PN-NanoVelcro chip at different electrospinning time points. (B) The cell capture efficiency of PN-NanoVelcro chip in different electrospinning flow rates. (C) The distribution of cell in different microchannels at flow rate of $1.0 \mathrm{~mL} / \mathrm{h}$. (D) Comparison of the capture performance between PN-NanoVelcro chip and four different controls: I) PN-NanoVelcro chip with herringbone and anti-EpCAM; 2) PN-NanoVelcro chip without herringbone structure; 3) anti-EpCAM-coated PLGA thin film with electrospinning; 4) PE-NanoVelcro chip without anti-EpCAM. (E) Capture efficiencies at different spiking cell numbers ranging from I0 to I,000/mL. (F) Capture efficiencies of different cell lines of NSCLC and WBCs.

Abbreviations: EpCAM, epithelial cell adhesion molecule; NSCLC, non-small cell lung cancer; PLGA, poly(lactic-co-glycolic acid); WBC, white blood cell.

blood. Consistent recovery rates were observed at various numbers of spiked cells as low as 10 cells/mL (Figure 2E). Various kinds of cell lines of NSCLC were used to prove that PN-NanoVelcro chips could specifically capture cancer cells instead of WBCs (Figure 2F).

\section{Immunostaining for CTC identification}

In order to confirm the feasibility of three-color immunostaining method for differentiating CTCs from WBCs, NCIH1975 was chosen as a positive control. The CK+/CD45-/ DAPI+ cells were defined as CTC candidates, while the $\mathrm{CK}-/ \mathrm{CD} 45+/ \mathrm{DAPI}+$ cells were considered as WBCs, and their locations were recorded and double-checked under $40 \times$ objectives for cellular morphology (Figure 3).

\section{EGFR mutations presented in both primary tissues and CTCs}

The mutational landscapes showed the specific mutation sites in primary tissues and CTCs of the 72 NSCLC patients, including 19-Del, L858R, T790M, 20-Ins, G719X, S768I and L861Q (Figure 4A). On comparing the mutation sites between primary tissues and CTCs, we found that the mutation sites were almost consistent to some extent. Also, the Venn diagrams demonstrated that EGFR mutations in
CTCs were almost in concordance with those in primary tissues (Figure 4B).

\section{EGFR L858R (exon 2I) and T790M (exon 20) sequencing of tissues and CTCs}

PCR results also showed that mutations in both exon 20 and exon 21 could be detected in the primary tissue and CTCs, following which the ARMS-PCR results further confirmed L858R mutation in exon 21 and T790M mutation in exon 20 in the CTCs. Finally, Sanger sequencing technology was implemented to confirm the specific mutation sites of L858R and T790M. As shown in the results, we can primarily come to a conclusion that EGFR mutations found in primary tissues could also be found in CTCs, which may provide us with a new insight that CTC detection could be a substitute for primary tissue biopsy (Figure 5).

\section{The correlation of T790M mutation and gefitinib-induced acquired resistance}

As shown in the CT results (Figure 6A) of primary tumor, the primary tumor appeared to shrink gradually after gefitinib treatment for 132 days. However, gefitinib seemed to be less effective after 193 days, and the tumor progressed after 251 days. CTCs were analyzed by ARMS-PCR at the same 


\section{NCI-H1975/WBC}

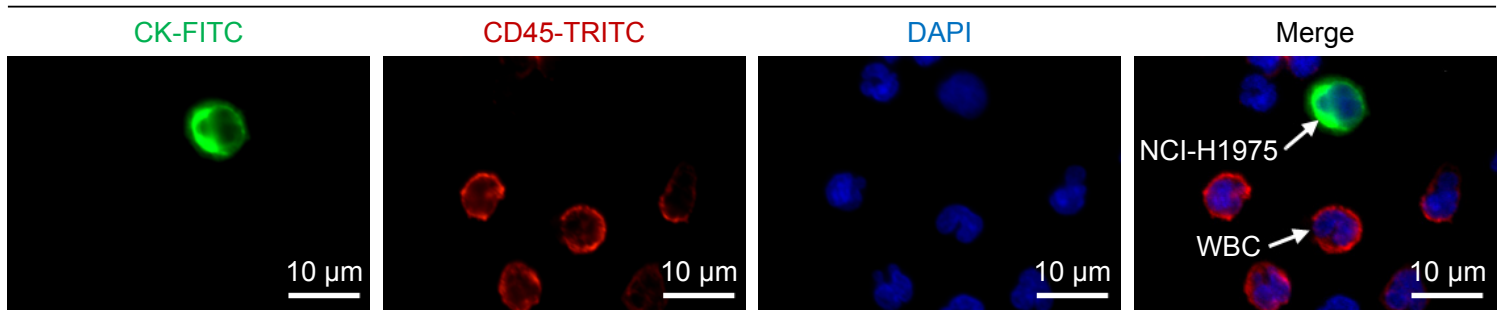

\section{CTC/WBC}

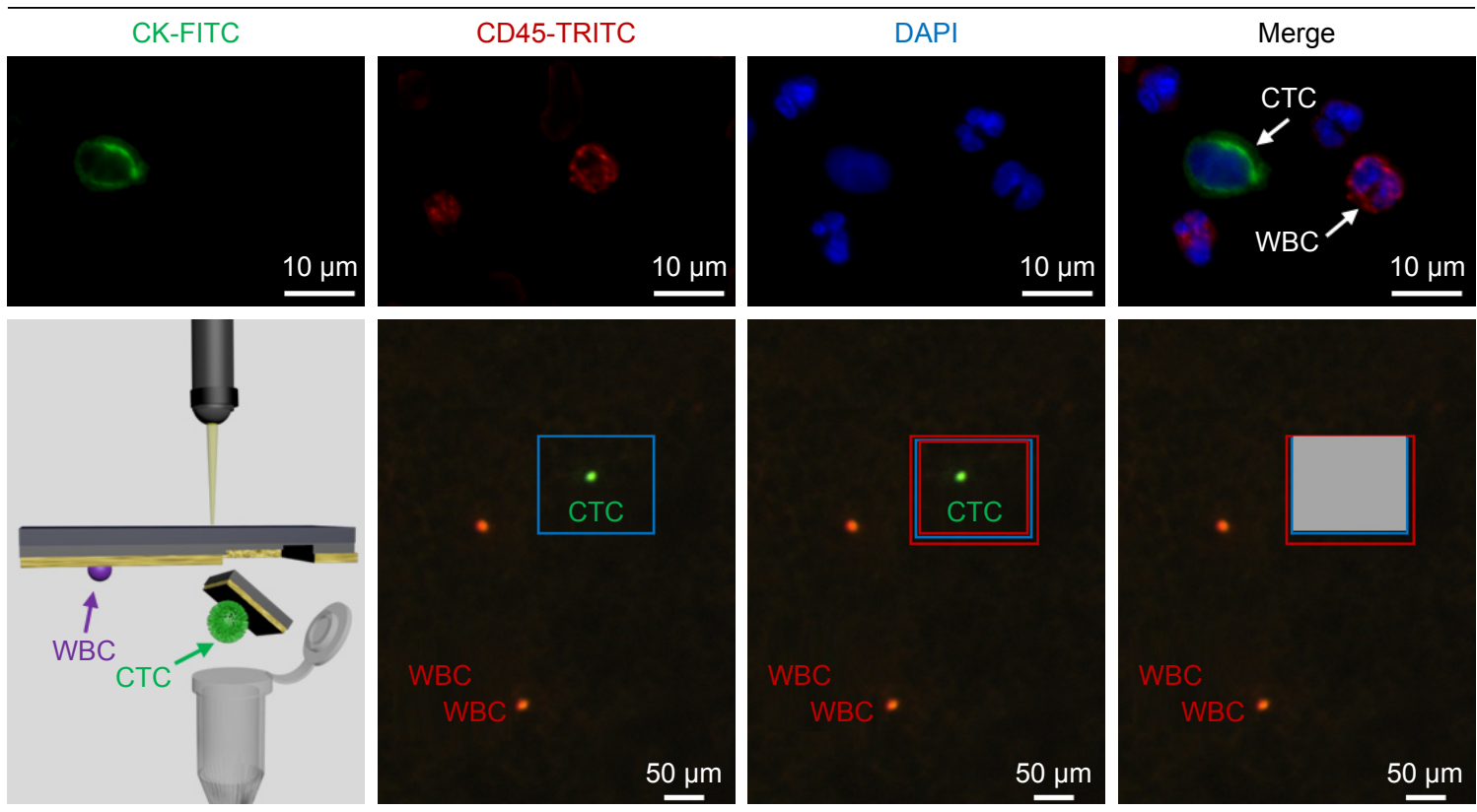

Figure 3 Identification of CTC, based on a three-color immunostaining using FITC-conjugated anti-CK, TRICT-conjugated anti-CD45 (a marker of WBC) and DAPI (nuclear specific).

Notes: $\mathrm{NCl}-\mathrm{HI} 975$ cells spiked in normal blood were used as a positive control. Isolating a single CTC was performed through laser microdissection after confirming the location by immunostaining.

Abbreviations: CTC, circulating tumor cell; DAPI, 4,6-diamino-I2-phenyl indole; FITC, fluorescein isothiocyanate; TRICT, tetramethylrhodamine; WBC, white blood cell.

time during the time course. According to the AMRS-PCR results (Figure 6B), we discovered that only L858R mutation could be found in previous successful gefitinib treatment, while both L858R and T790M appeared in CTCs of NSCLC patients with acquired resistance. Comparing the results of ARMS-PCR to those of CT images, T790M mutation could be detected long before tumor progression, which means that CTC analysis can provide possibility for early prognosis.

\section{Discussion}

Lung cancer, the main cause of cancer-related death worldwide, has been a threat to health that could not be ignored. ${ }^{25}$ Currently, tumor tissues used for EGFR mutations analysis are acquired through surgery. However, tumor tissues are unavailable in some cases, so a substitute method of biopsy is in great need. Taking the advantages into consideration, CTC detection is recognized to be a better choice not only for its acquisition without invasiveness, but also for its possibility to be conducted throughout the course of disease.

Our study aimed to develop a new method to capture CTCs with high efficiency and purity and implement genetic analysis of the isolated single CTC throughout the time course of disease. The evaluation results of optimization and validation of modified PN-NanoVelcro chip showed that the capture efficiencies of different cell lines of NSCLC could reach about $80 \%$, which means that PN-NanoVelcro chip can be an effective method for capturing CTCs. Apart from developing a new approach to capture CTCs, we also found out the concordance of the EGFR mutation sites between tumor tissues and CTCs acquired from 72 NSCLC patients. In addition, the correlation between specific EGFR mutation sites and gefitinib-induced acquired assistance has also been preliminarily clarified, which may disclose the reasons for discouraging gefitinib treatment to some extent. 
A
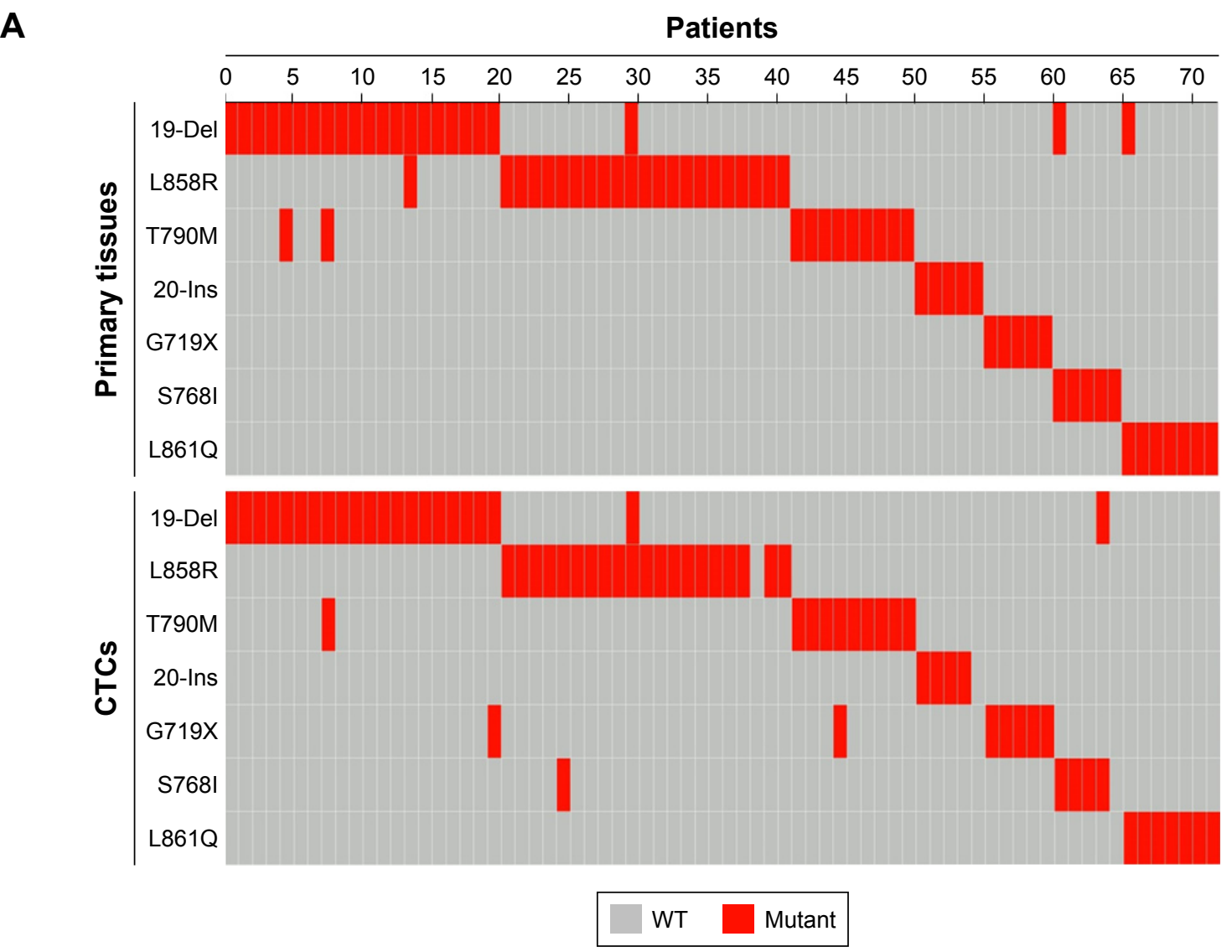

B

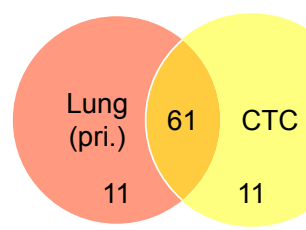

20-Ins

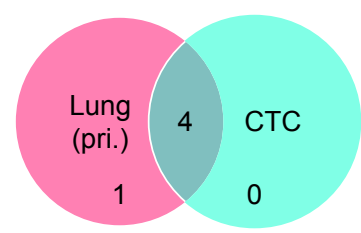

19-Del

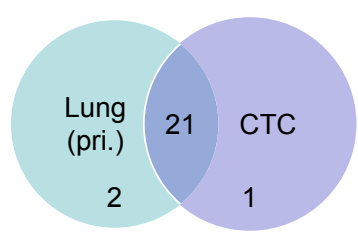

G719X

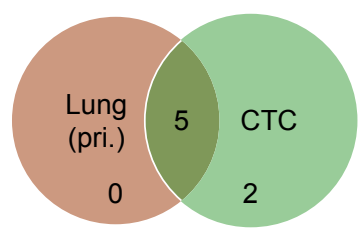

L858R

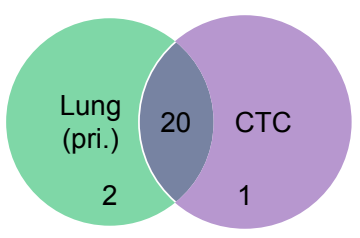

S768I

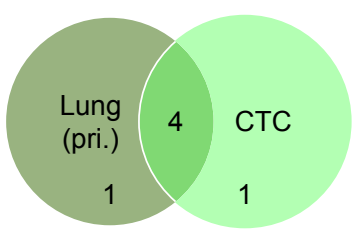

T790M

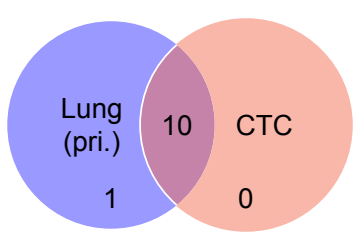

L861Q

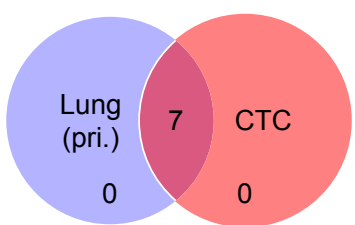

Figure 4 Concordance of EGFR mutation sites detected in primary tissues and CTCs from NSCLC patients.

Notes: (A) Landscape of somatic mutations detected in primary tissues and CTCs from 72 patients with NSCLC. Seven EGFR alterations were detected by targeted sequencing and the results were computed in the mutational landscape. (B) The Venn diagrams were used to summarize the similarity of EGFR mutations in primary tissues and CTCs from 72 NSCLC patients.

Abbreviations: CTC, circulating tumor cell; EGFR, epidermal growth factor receptor; NSCLC, non-small cell lung cancer; pri., primary tissue; WT, wild type.

As known to all, EGFR mutation in the tumor tissues has a close relationship with NSCLC. ${ }^{26}$ With a further study of the EGFR mutations in NSCLC, specific mutation sites with different functions have been discovered. Numerous researches have demonstrated that NSCLC patients with mutation sites such as 19-Del, L858R, 20-Ins, G719X, S768I and L861Q seemed to be sensitive to gefitinib and could undergo successful treatment. ${ }^{27}$ According to the mutational spectrum in our study, we discovered similar mutation sites in primary tissues and CTCs, which makes observation of timely tumor progression possible.

As reported in clinical researches, NSCLC patients cannot benefit from EGFR-TKIs all along, as the majority of patients with EGFR mutation would eventually develop 
A
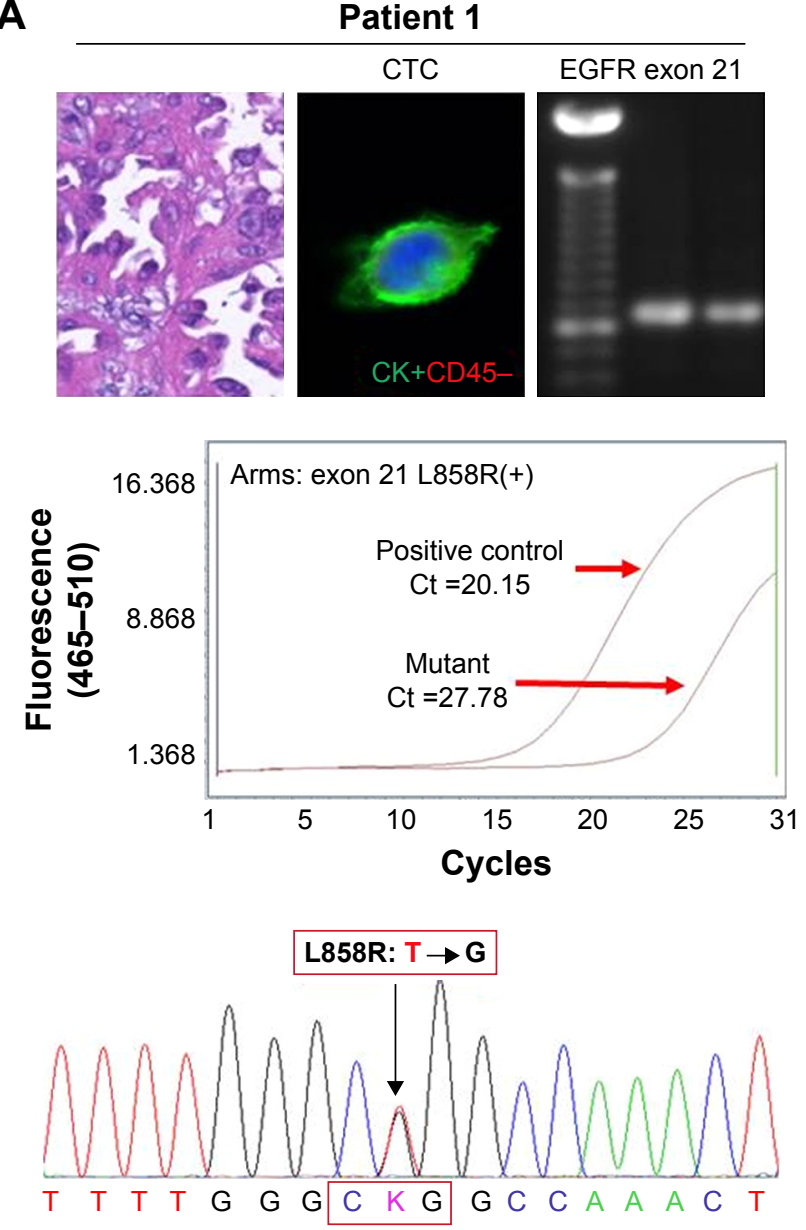

B
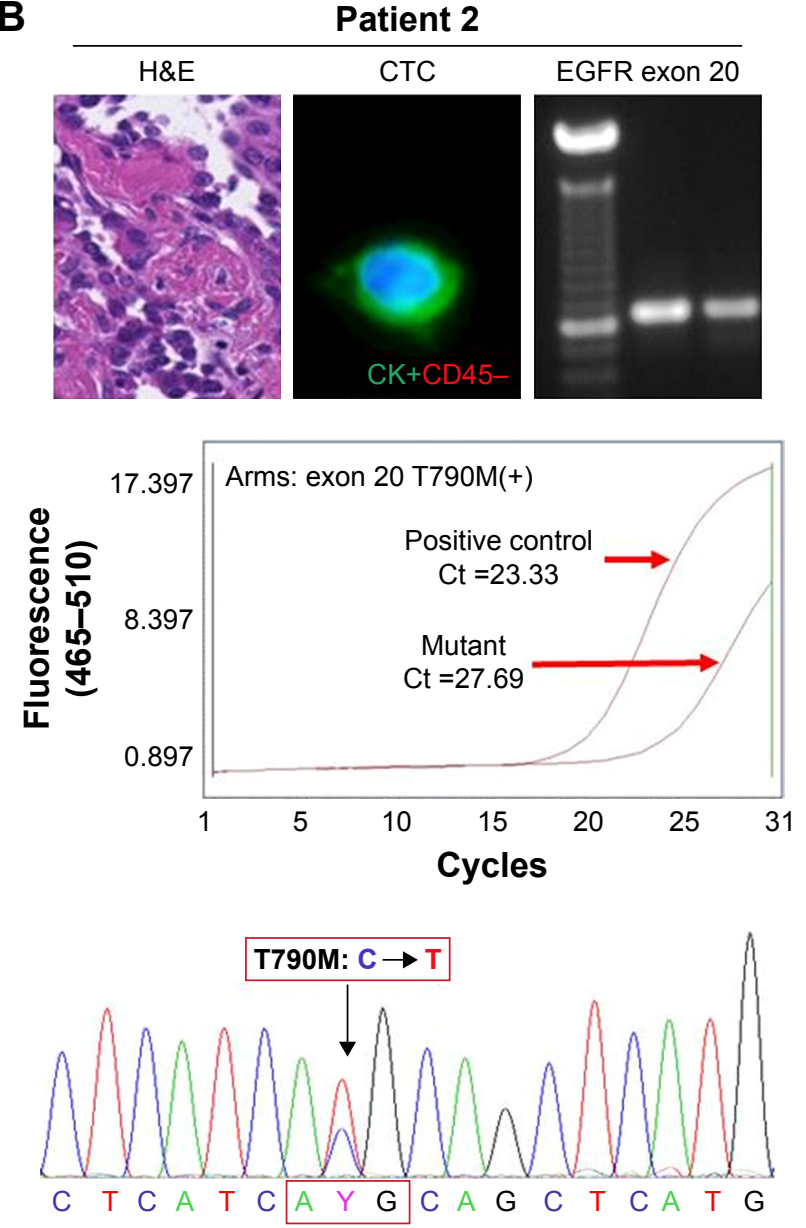

Figure 5 EGFR L858R (exon 21) and T790M (exon 20) sequencing of primary tissues and CTCs.

Notes: (A) PCR of exon 21 was successfully performed and a band of proper size was found with $2 \%$ gel electrophoresis. ARM-PCR and Sanger sequencing were used to detect the mutation sites in L858R. (B) PCR of exon 20 was successfully performed and a band of proper size was found with $2 \%$ gel electrophoresis. ARM-PCR and Sanger sequencing were used to detect the mutation sites in T790M.

Abbreviations: ARM-PCR, amplification refractory mutation system PCR; CTCs, circulating tumor cells; EGFR, epidermal growth factor receptor; PCR, polymerase chain reaction.

acquired resistance after $12-24$ months of treatment. ${ }^{28}$ T790M, a mutation of exon 20 in EGFR, has been reported to be closely related to acquired resistance induced by gefitinib. ${ }^{29}$ Some researchers have found that T790M mutation reduced the affinity to EGFR-TKIs, which may be the cause of gefitinib-induced acquired resistance. ${ }^{30}$ As shown in the CT results and ARMS-PCR results of L858R and T790M mutations during the time course of the disease, it is clear that NSCLC patients with L858R mutation can acquire obvious efficacy after initial treatment with gefitinib.
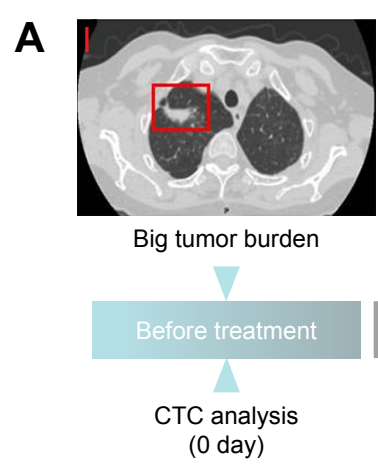

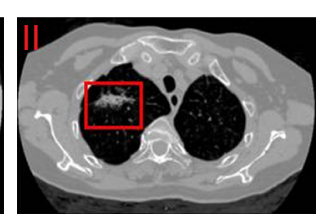

Tumor shrinkage $\nabla$

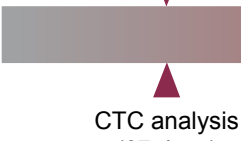

(67 days)

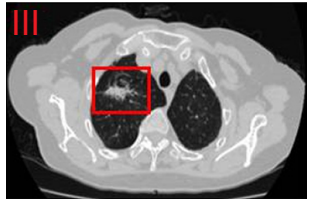

Tumor shrinkage

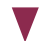

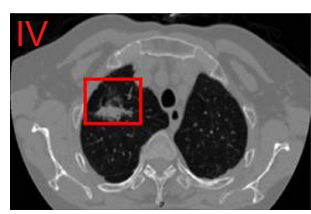

Tumor stabilization

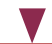

Gefitinib treatment

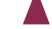

CTC analysis

(132 days)

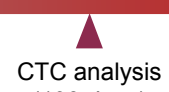

(193 days)

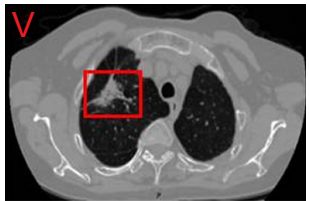

Tumor progression
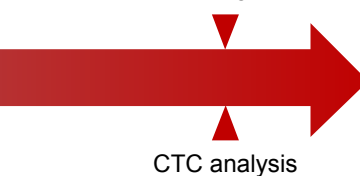

CTC analysis

(251 days)

Figure 6 (Continued) 


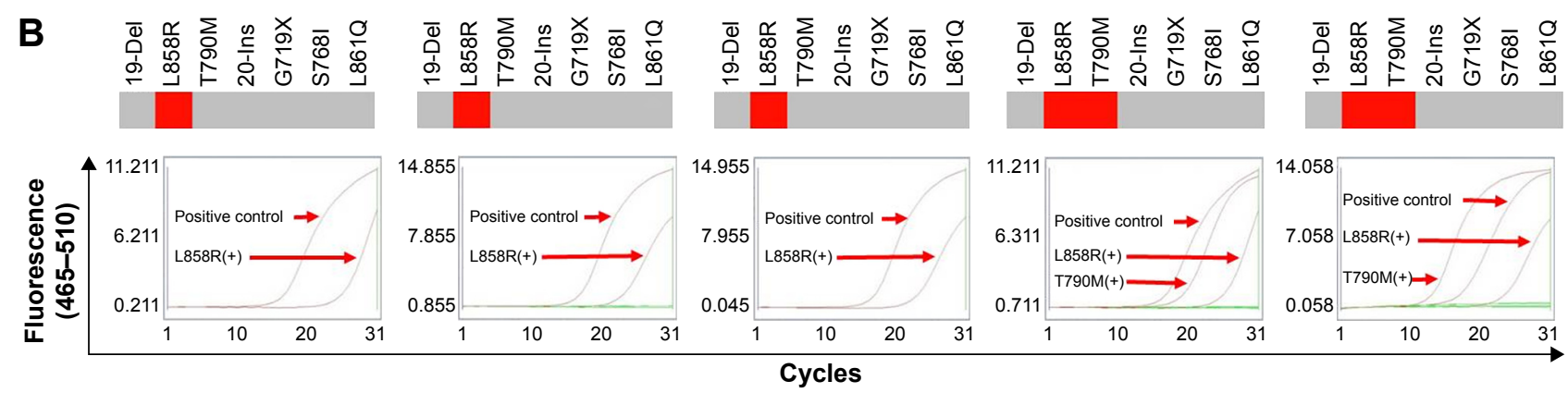

Figure 6 The relationship between acquired resistance induced by continuous gefitinib treatment and EGFR mutation.

Notes: (A) Course of the disease with CT images and treatment history and driver mutation. (B) L858R and T790M mutation detection via ARMS-PCR.

Abbreviations: ARMS-PCR, amplification refractory mutation system polymerase chain reaction; CT, computed tomography; CTC, circulating tumor cell; EGFR, epidermal growth factor receptor.

However, gefitinib became less effective gradually or even lost its effect at last. Mutation analysis of tumor has shown that not only LR858 mutation but also T790M mutation appears in patients with acquired resistance. In general clinical application, L858R and T790M mutations are implemented through tumor tissues obtained from surgery. Compared with traditional tissue biopsy, CTC detection can be a better alternative to monitor patients' state of illness for its noninvasive acquisition and simple procedure throughout the course of diseases. With our further research of CTCs in the following study, the mechanisms of NSCLC with and without EGFR mutations could be further clarified. Also, we would develop a technique to culture the isolated CTCs in vitro in the future, with an aim to find a suitable drug therapy for NSCLC patients and lay solid foundation for the realization of individual treatments.

\section{Acknowledgments}

This work was supported by grants from the National Natural Science Foundation of China (30900650, 81372501, 81572260 and 81773299), the Guangdong Natural Science Foundation (2011B031800025, S2012010008378, 2015A030313036 and 2015A030313036) and the Guangzhou and Guangdong/Guangzhou Science and Technology Planning Program (2014J4100132, 2012B031800115, 2013B02180021, 2015A020214010, 2016A020215055, 201704020094, 16ykjc08 and 2015ykzd07).

\section{Disclosure}

The authors report no conflicts of interest in this work.

\section{References}

1. Cheng TY, Cramb SM, Baade PD, Youlden DR, Nwogu C, Reid ME. The International epidemiology of lung cancer: latest trends, disparities, and tumor characteristics. J Thorac Oncol. 2016;11(10):1653-1671.
2. Roca E, Gurizzan C, Amoroso V, Vermi W, Ferrari V, Berruti A Outcome of patients with lung adenocarcinoma with transformation to small-cell lung cancer following tyrosine kinase inhibitors treatment: a systematic review and pooled analysis. Cancer Treat Rev. 2017;59: $117-122$.

3. Torre LA, Siegel RL, Jemal A. Lung cancer statistics. Adv Exp Med Biol. 2016;893:1-19.

4. Zhang Y, Lv Y, Niu Y, Su H, Feng A. Role of circulating tumor cell (CTC) monitoring in evaluating prognosis of triple-negative breast cancer patients in China. Med Sci Monit. 2017;23:3071-3079.

5. Xu T, Shen G, Cheng M, Xu W, Shen G, Hu S. Clinicopathological and prognostic significance of circulating tumor cells in patients with lung cancer: a meta-analysis. Oncotarget. 2017;8(37):62524-62536.

6. Wu W, Zhang Z, Gao XH, et al. Clinical significance of detecting circulating tumor cells in colorectal cancer using subtraction enrichment and immunostaining-fluorescence in situ hybridization (SE-iFISH). Oncotarget. 2017;8(13):21639-21649.

7. Liu W, Yin B, Wang X, et al. Circulating tumor cells in prostate cancer: precision diagnosis and therapy. Oncol Lett. 2017;14(2):1223-1232.

8. Yang D, Wang L, Tian X. Application of circulating tumor cells scope technique on circulating tumor cell research. Mol Cell Ther. 2014;2:8.

9. Kallergi G, Politaki E, Alkahtani S, Stournaras C, Georgoulias V. Evaluation of isolation methods for circulating tumor cells (CTCs). Cell Physiol Biochem. 2016;40(3-4):411-419.

10. Vila A, Abal M, Muinelo-Romay L, et al. EGFR-based immunoisolation as a recovery target for low-EpCAM CTC subpopulation. PLoS One. 2016;11(10):e0163705.

11. Lee M, Kim EJ, Cho Y, et al. Predictive value of circulating tumor cells (CTCs) captured by microfluidic device in patients with epithelial ovarian cancer. Gynecol Oncol. 2017;145(2):361-365.

12. Kang YT, Doh I, Byun J, Chang HJ, Cho YH. Label-free rapid viable enrichment of circulating tumor cell by photosensitive polymer-based microfilter device. Theranostics. 2017;7(13):3179-3191.

13. Zhou MD, Hao S, Williams AJ, et al. Separable bilayer microfiltration device for viable label-free enrichment of circulating tumour cells. Sci Rep. 2014;4:7392.

14. Ran R, Li L, Wang M, Wang S, Zheng Z, Lin PP. Determination of EGFR mutations in single cells microdissected from enriched lung tumor cells in peripheral blood. Anal Bioanal Chem. 2013;405(23):7377-7382.

15. Li YQ, Liu YS, Ying XW, et al. Lentivirus-mediated disintegrin and metalloproteinase 17 RNA interference reversed the acquired resistance to gefitinib in lung adenocarcinoma cells in vitro. Biotechnol Prog. Epub 2017 Sep 27.

16. Mitsudomi T, Kosaka T, Yatabe Y. Biological and clinical implications of EGFR mutations in lung cancer. Int J Clin Oncol. 2006;11(3):190-198.

17. Lee DH, Srimuninnimit V, Cheng R, Wang X, Orlando M. Epidermal growth factor receptor mutation status in the treatment of non-small cell lung cancer: lessons learned. Cancer Res Treat. 2015;47(4):549-554. 
18. Lynch TJ, Bell DW, Sordella R, et al. Activating mutations in the epidermal growth factor receptor underlying responsiveness of nonsmall-cell lung cancer to gefitinib. N Engl J Med. 2004;350(21): 2129-2139.

19. Xu M, Xie Y, Ni S, Liu H. The latest therapeutic strategies after resistance to first generation epidermal growth factor receptor tyrosine kinase inhibitors (EGFR TKIs) in patients with non-small cell lung cancer (NSCLC). Ann Transl Med. 2015;3(7):96.

20. Liang Z, Cheng Y, Chen Y, et al. EGFR T790M ctDNA testing platforms and their role as companion diagnostics: correlation with clinical outcomes to EGFR-TKIs. Cancer Lett. 2017;403:186-194.

21. Takamochi K, Oh S, Matsunaga T, Suzuki K. Prognostic impacts of EGFR mutation status and subtype in patients with surgically resected lung adenocarcinoma. J Thorac Cardiovasc Surg. 2017;154(5): 1768.e1-1774.e1.

22. Court CM, Ankeny JS, Sho S, et al. Reality of single circulating tumor cell sequencing for molecular diagnostics in pancreatic cancer. $J \mathrm{Mol}$ Diagn. 2016;18(5):688-696.

23. Schneck H, Blassl C, Meier-Stiegen F, et al. Analysing the mutational status of PIK3CA in circulating tumor cells from metastatic breast cancer patients. Mol Oncol. 2013;7(5):976-986.
24. Zhang C, Wei B, Li P, et al. Prognostic value of plasma EGFR ctDNA in NSCLC patients treated with EGFR-TKIs. PLoS One. 2017;12(3): e0173524.

25. Siegel R, Ma J, Zou Z, Jemal A. Cancer statistics, 2014. CA Cancer J Clin. 2014;64(1):9-29.

26. Lin CC, Huang WL, Wei F, Su WC, Wong DT. Emerging platforms using liquid biopsy to detect EGFR mutations in lung cancer. Expert Rev Mol Diagn. 2015;15(11):1427-1440.

27. Furuyama K, Harada T, Iwama E, et al. Sensitivity and kinase activity of epidermal growth factor receptor (EGFR) exon 19 and others to EGFR-tyrosine kinase inhibitors. Cancer Sci. 2013;104(5):584-589.

28. Cappuzzo F, Morabito A, Normanno N, et al. Efficacy and safety of rechallenge treatment with gefitinib in patients with advanced non-small cell lung cancer. Lung Cancer. 2016;99:31-37.

29. Li W, Ren S, Li J, et al. T790M mutation is associated with better efficacy of treatment beyond progression with EGFR-TKI in advanced NSCLC patients. Lung Cancer. 2014;84(3):295-300.

30. Giaccone G. EGFR point mutation confers resistance to gefitinib in a patient with non-small-cell lung cancer. Nat Clin Pract Oncol. 2005;2(6): 296-297.
International Journal of Nanomedicine

\section{Publish your work in this journal}

The International Journal of Nanomedicine is an international, peerreviewed journal focusing on the application of nanotechnology in diagnostics, therapeutics, and drug delivery systems throughout the biomedical field. This journal is indexed on PubMed Central, MedLine, CAS, SciSearch $®$, Current Contents ${ }^{\circledR} /$ Clinical Medicine,

\section{Dovepress}

Journal Citation Reports/Science Edition, EMBase, Scopus and the Elsevier Bibliographic databases. The manuscript management system is completely online and includes a very quick and fair peer-review system, which is all easy to use. Visit http://www.dovepress.com/ testimonials.php to read real quotes from published authors. 